\title{
Nuevo modelo para la predicción de la microestructura final y los parámetros de recristalización dinámica de materiales metálicos policristalinos. Aplicación a la aleación de magnesio AZ31
}

\author{
I. Rieiro*, M.T. Larrea**, V. Gutiérrez*,V. Triviño*, M. Carsí** y O.A. Ruano**
}

\begin{abstract}
Resumen
Se ha partido de una nueva ecuación constitutiva capaz de reproducir las curvas tensión-deformación para cada velocidad de deformación y temperatura. Aplicando esta ecuación se pueden obtener los parámetros característicos de la recristalización dinámica para cada una de las curvas de fluencia. En este trabajo se ha hecho un estudio experimental de los tamaños de grano finales, sus distribuciones de frecuencia y fracciones de área que, junto con los parámetros característicos de la recristalización dinámica, han permitido establecer modelos predictivos para las condiciones finales de microestructura de materiales metálicos policristalinos. El método se ha aplicado a la aleación de magnesio AZ31 deformada en caliente. Se predicen tanto valores promedio de tamaños de grano final como distribuciones de fracciones de área y de frecuencia de los mismos. También, se predicen los parámetros característicos de la recristalización dinámica del material que constituyen elementos de control del proceso de deformación.
\end{abstract}

\section{New model for the prediction of the final microstructure and the dynamic recrystallization parameters of polycrystalline metallic materials. Application to AZ31 magnesium alloy}

\begin{abstract}
The study starts from a new constitutive equation able to reproduce the stress-strain curves for each strain rate and temperature. Using this equation, the characteristic parameters of dynamic recrystallization for each of the flow curves can be obtained. An experimental study of final grain sizes, their frequency and distribution and area fractions has been made. These parameters together with those mentioned above allow establishing predictive models for the final microstructure conditions of polycrystalline metallic materials. These models have been applied to the hot deformation of AZ31 magnesium alloy. Mean final grain sizes, area fraction distributions and their frequency are predicted. In addition, the characteristic dynamic recrystallization parameters of the material that constitute elements of control of the deformation process are also predicted.
\end{abstract}

Keywords

Modelization; Plastic flow; Dynamic recrystallization; Magnesium alloys.

\section{INTRODUCCIÓN}

Para poder predecir las condiciones microestructurales finales de un material metálico policristalino y los parámetros que controlan su deformación plástica en caliente es preciso realizar las etapas siguientes: obtención de la ecuación constitutiva, determinación de los parámetros de la recristalización dinámica y descripción de la estadística de las microestructuras finales. La calidad de los resultados de cada una de las etapas secuenciales depende de la adecuada base física y de los tratamientos matemáticos numéricos, algorítmicos y estadísticos aplicados. El esquema global seguido en este trabajo se encuentra descrito en la figura 1.

En la primera etapa, se ha utilizado una nueva ecuación constitutiva para reproducir las curvas tensión, $\sigma$, - deformación, $\varepsilon$, para cada velocidad de deformación, $\dot{\varepsilon}$, y temperatura $\mathrm{T}^{[1]}$. Dicha ecuación, basada en la ecuación de Garofalo, tiene la forma:

\footnotetext{
* Dpto. Matemáticas, Universidad de Castilla-La Mancha, Avda. Carlos III s/n, 45071 Toledo. e-mail: Ignacio.Rieiro@uclm.es.

** CENIM-CSIC, Av. Gregorio del Amo, 8, 28040 Madrid.e-mail: ruano@cenim.csic.es.
} 


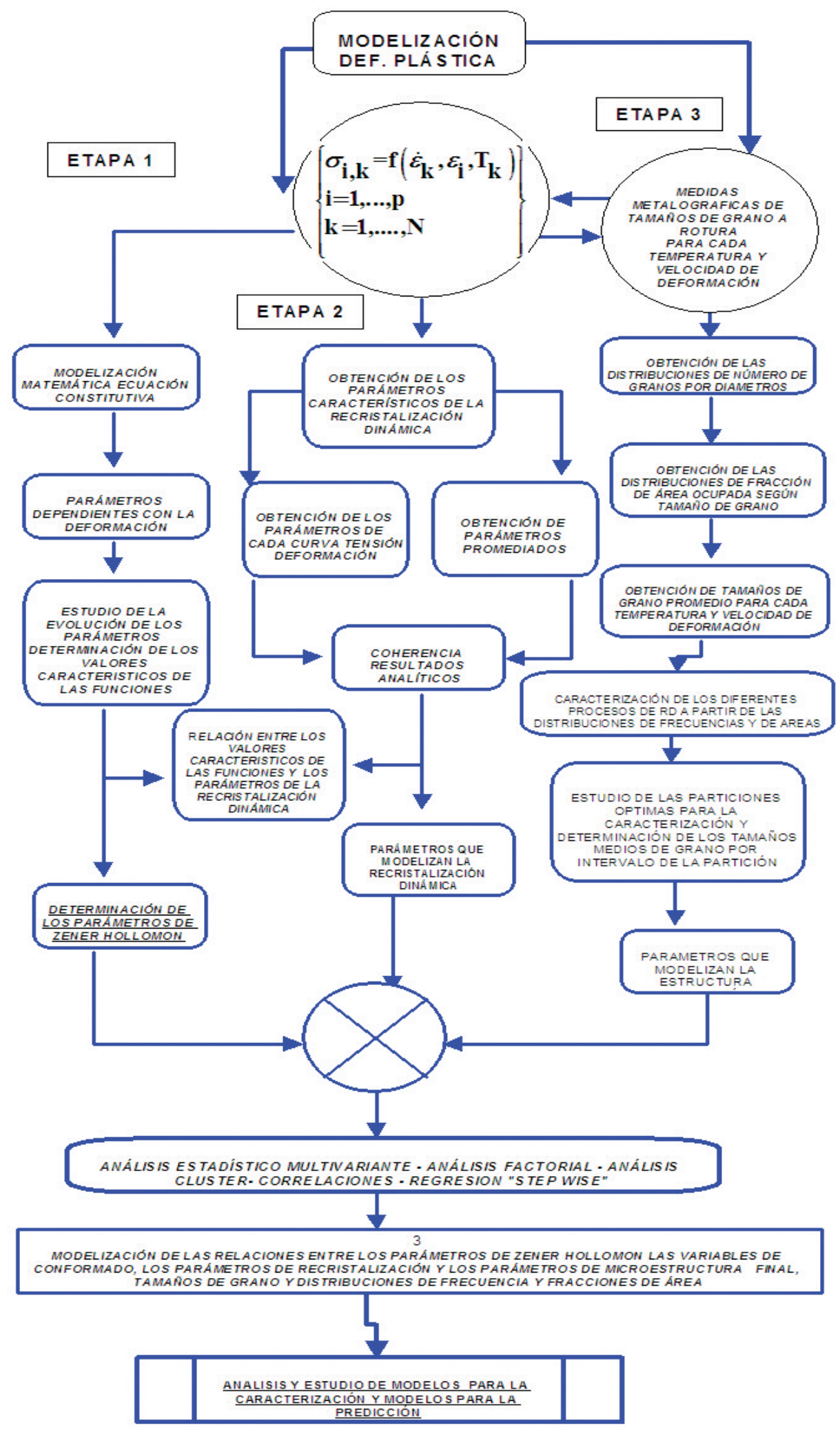

Figura 1. Descripción abreviada del proceso de modelización múltiple empleado. Detalle de las tres etapas de medida y construcción de parámetros representativos de la fluencia plástica y de los procesos involucrados, recristalización dinámica, microestructura y su evolución.

Figure 1. Short description of the used multiple modellization process. Detail of the three measurement stages and construction of the representative parameters for creep and processes involved, dynamic recrystallization, microstructure and its evolution. 


$$
\sigma(\varepsilon)=\frac{\ln \left(Z^{*}(\dot{\varepsilon}, T, \varepsilon)+\sqrt{\left(Z^{*}(\dot{\varepsilon}, T, \varepsilon)\right)^{2}+1}\right.}{\alpha(\varepsilon)}
$$

donde,

$$
Z^{*}(\dot{\varepsilon}, T, \varepsilon)=\left(\dot{\varepsilon} \cdot e^{Q(\varepsilon)} / A(\varepsilon)\right)^{\frac{1}{n(\varepsilon)}}
$$

El parámetro $Z^{*}$, es el parámetro de ZenerHollomon, reducido mediante $\mathrm{A}(\varepsilon)$ y dependiente de la deformación. Las variables termodinámicas son las usuales, ya definidas, y los parámetros de la ecuación constitutiva son $\{\mathrm{A}(\varepsilon), \mathrm{Q}(\varepsilon), \mathrm{n}(\varepsilon), \alpha(\varepsilon)\}$, los usuales de la ecuación de Garofalo ${ }^{[1]}$, pero dependientes de la deformación y con sentido diferente detallado en anteriores trabajos ${ }^{[2}$ y 3$]$. Al proceso de obtención de los parámetros óptimos que definen la ecuación (1) se han aplicado métodos numéricos no lineales, de segundo orden de aproximación. Esto se ha hecho a partir de la reducción de datos y de la

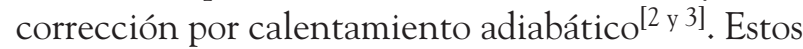
métodos, RCR y Newton, han sido descritos previamente $^{[2,4 \text { y } 5]}$.

Como resultado final del establecimiento de la ecuación constitutiva se obtienen relaciones funcionales dependientes de la deformación para los parámetros que la caracterizan. Las expresiones evolutivas de $\{\mathrm{A}(\varepsilon), \mathrm{Q}(\varepsilon), \mathrm{n}(\varepsilon), \alpha(\varepsilon)\}$, permiten asociar los puntos singulares de las formas funcionales con los parámetros de la recristalización dinámica ${ }^{[1]}$.

En la segunda etapa, se han implementado dos métodos concurrentes ${ }^{[6}$ y 7$]$ para determinar los parámetros característicos de la recristalización dinámica (RD), que son los de deformación y tensión de inicio de formación de subgranos $\left(\varepsilon_{\text {is }}, \sigma_{\text {is }}\right)$, los de inicio de $\operatorname{RD}\left(\varepsilon_{\text {idrx }}, \sigma_{\text {idrx }}\right)$, los de recristalización para el \% de volumen recristalizado $\left(\varepsilon_{50}, \sigma_{50}\right)$, los de máximo $\left(\varepsilon_{\mathrm{p}}, \sigma_{\mathrm{p}}\right)$, los de estado estacionario $\left(\varepsilon_{\mathrm{ss}}, \sigma_{\mathrm{ss}}\right)$ y los de saturación $\left(\varepsilon_{\text {sat }}, \sigma_{\text {sat }}\right)$, todos ellos, obtenidos a T y $\dot{\varepsilon}$ constantes. El primer método de determinación de los parámetros de RD está basado en los trabajos tradicionales de McQueen, Galiyev y Poliak ${ }^{[8-10]}$, mejorados con un método no lineal para la obtención de la deformación a la cual se alcanza el 50 \% de la fracción recristalizada, dependiendo de la temperatura y la velocidad de deformación. Los parámetros de la $\mathrm{RD}$ pueden obtenerse a partir del endurecimiento por deformación o sensibilidad a la deformación, $\theta$

$$
\theta(\sigma)=\frac{\partial \sigma}{\partial \varepsilon}
$$

El inicio de la formación de subgranos se producirá en el mínimo de la curva . A partir de este punto, la curva $S(\sigma)=-\partial \theta / \partial \sigma$ presenta una evolución lineal y en el punto donde se produce la salida del comportamiento lineal es donde se asume el inicio de RD. Posteriormente, el máximo de tensión, $\sigma_{\mathrm{p}}$, se alcanza para los valores de tensión y deformación para los que $\theta=0$. Se ha determinado la deformación de recristalización para el $50 \%$ de volumen recristalizado a partir de una ecuación constitutiva basada en el modelo de Avrami ${ }^{[7]}$ :

$$
\sigma=\sigma_{p}-\left(\sigma_{p}-\sigma_{s s}\right)\left\{1-\exp \left[-\ln 2\left(\frac{\varepsilon-\varepsilon_{p}}{\varepsilon_{50}-\varepsilon_{p}}\right)^{k}\right]\right\}
$$

donde, $\mathrm{k}$ es una constante.

El segundo método se basa en modelos no lineales ad-hoc, a partir de la ecuación constitutiva y de la evolución de los parámetros $\{\mathrm{A}(\varepsilon), \alpha(\varepsilon)\}^{[11]}$, de donde se obtienen los valores de deformación a los cuales se alcanza el $50 \%$ de la fracción de volumen recristalizado y que permite predecir la evolución del tamaño de grano del material, siempre para valores promedio de los rangos de las variables de trabajo. La fracción de volumen recristalizada viene dada por

$$
X(\varepsilon)=\left(\frac{a}{1+b}\right) /\left(1+b e^{-c \varepsilon}\right)
$$

donde, a, b y c se obtienen mediante la optimización del modelo no lineal sigmoidal:

$$
\alpha(\varepsilon)=\alpha_{0} \cdot\left(1-\frac{a}{1+b \cdot e^{-c \varepsilon}}\right)
$$

En la tercera etapa, se ha desarrollado un método experimental de tratamiento de microestructuras ${ }^{[12]}$ con una alta calidad en la detección y contaje de los granos, a cuyos resultados se ha aplicado un método probabilístico nuevo, para el cálculo de tamaños medios de grano. Este método está asociado a las distribuciones de frecuencias de los granos, junto con un pesado probabilístico a partir de su área. Este método se ha calibrado con los tradicionales de las normas UNE y ASTM de intersección lineal y de caracterización de micrografías estandarizadas. 
Es importante hacer un resumen de los parámetros obtenidos en la tercera etapa. Definida el área de un grano como $s_{i}\{i=1, . ., N\}$, se define la longitud de grano como $\mathrm{l}_{\mathrm{i}}=\mathrm{k}_{\mathrm{G}} \cdot \sqrt{\mathrm{s}_{\mathrm{i}}}$. La constante $\mathrm{k}_{\mathrm{G}}$ es de índole geométrica. El valor promedio de las longitudes es $\Phi_{1}$. Se definen tres zonas de longitud representativa de grano, $[0,15) \mu \mathrm{m},[15,40) \mu \mathrm{m}$ y $(40,80]$ $\mu \mathrm{m}$, calculándose los valores promedio de las longitudes en dichas zonas, siendo estos valores $\Phi_{\mathrm{A} 1}, \Phi_{\mathrm{A} 2}$ y $\Phi_{\mathrm{A} 3}$. Si el área total de los granos de cada zona es $\mathrm{S}_{\mathrm{A} 1}, \mathrm{~S}_{\mathrm{A} 2}$ y $\mathrm{S}_{\mathrm{A} 3}$, entonces, la probabilidad de aparición de un grano, cuyo tamaño se encuentre en cada zona, es: $\mathrm{P}_{\mathrm{Ai}}\{\mathrm{i}=1,2,3\}$, siendo $\mathrm{P}_{\mathrm{Ai}}=\mathrm{S}_{\mathrm{Ai}} / \mathrm{S}_{\mathrm{T}}$ y $\mathrm{S}_{\mathrm{T}}$ el área total.

El tamaño de grano medio es $\Phi_{2}=\sum_{i=1}^{\mathrm{i}=3} \Phi_{\mathrm{Ai}} \cdot P_{\mathrm{Ai}}$. Se definen cuatro zonas de longitud de grano $[0,10) \mu \mathrm{m}$, $[10,30) \mu \mathrm{m},[30,50) \mu \mathrm{m}$ y $[50,80] \mu \mathrm{m}$, partición $\mathrm{P}(4)$, y con la misma nomenclatura anterior $\Phi_{3}=\sum_{i=1}^{i=4} \Phi_{A i}$. $P_{A i}$

Cuando la probabilidad de que un grano sea medido es $\Pi_{\mathrm{i}}=\mathrm{s}_{\mathrm{i}} / \mathrm{S}_{\mathrm{T}}$, entonces el tamaño de grano medio es $\Phi_{4}=\sum_{\mathrm{i}=1}^{\mathrm{i}=\mathrm{N}} \Phi_{\mathrm{A} i} \cdot P_{\mathrm{Ai}}$. Para establecer la distribución de frecuencias se ha usado una partición del intervalo $[0,80] \mu \mathrm{m}$, en subintervalos de amplitud $5 \mu \mathrm{m}$. En cada uno, $\mathrm{k}$, se ha medido el número de granos, $\mathrm{n}_{\mathrm{k}}$. La frecuencia relativa del subintervalo $k$ es $\mathrm{fr}_{\mathrm{k}}=\mathrm{n}_{\mathrm{k}} / \mathrm{n}_{\mathrm{T}}$, siendo $\mathrm{n}_{\mathrm{T}}$ el número de granos totales en el intervalo $[0,80] \mu \mathrm{m}$. La distribución de fracciones de área se define como $\mathrm{fra}_{\mathrm{k}}=\mathrm{s}_{\mathrm{k}} / \mathrm{S}_{\mathrm{T}}$, siendo $s_{\mathrm{k}}$ el área del subintervalo $\mathrm{k}$ del intervalo $[0,80] \mu \mathrm{m}$. El mismo procedimiento se ha llevado a cabo para la partición $\mathrm{P}(4)^{[12]}$. Esta partición es especialmente relevante porque segrega los tamaños de granos provenientes de diferentes mecanismos de RD ${ }^{[12]}$. En $[0,10) \mu \mathrm{m}$ se encuentran los granos de los primeros collares en el proceso de génesis de la RD por el mecanismo de collares (necklace), descrito por Ponge y Gottstein ${ }^{[13]}$. Para $[10,30) \mu \mathrm{m}$ se encuentran granos provenientes de la matriz original de extrusión y granos del primer collar en crecimiento. En el intervalo $[30,50) \mu \mathrm{m}$, se encuentran granos provenientes de segundos y terceros collares de procesos RD cíclicos sostenidos, correspondientes a RD primaria y algunos granos de RD secundaria, consecuencia de crecimiento anómalo de granos. Por último, en $[50,80] \mu \mathrm{m}$, fundamentalmente aparecen granos, fruto de RD

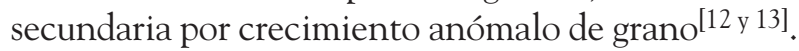

A partir de las etapas mencionadas, se han podido modelizar los parámetros que definen los procesos de $\mathrm{RD}$, así como las condiciones de la microestructura final de la aleación AZ31. Esto último se ha hecho tanto para el tamaño medio final de los granos como para la frecuencia de aparición de los granos y de la fracción de área ocupada por los mismos, según zonas diferenciales dadas por la partición P(4). Las predicciones se han realizado a partir de los parámetros dependientes de la deformación, de la ecuación constitutiva (Ec. (2)). Se han empleado diferentes herramientas estadísticas avanzadas para conseguir las predicciones con una alta precisión.

\section{MÉTODO EXPERIMENTAL}

Se partió de una aleación de magnesio AZ31 de composición $\mathrm{Mg}-3 \% \mathrm{Al}-1 \% \mathrm{Zn}$, suministrada en forma de barra extruida, de $\varnothing 1.000 \times 25 \mathrm{~mm}$, por Magnesium Elektron. Las probetas de torsión fueron de longitud útil de $50 \mathrm{~mm}$ y radio de $3 \mathrm{~mm}$. Los ensayos de torsión en caliente se llevaron a cabo en una máquina de ensayo SETARAM modelo $7 \mathrm{~N}-7 \mathrm{MN}$ [7 y 12$]$. Los ensayos se realizaron a $\mathrm{T} \in[300,410 \text { y } 450]^{\circ} \mathrm{C}$, y a $\dot{\varepsilon} \in[0,73,2$ y 8,73$] \mathrm{s}^{-1}$. Una vez ensayadas las probetas se cortaron a $5 \mathrm{~mm}$ de la zona de rotura, se embutieron longitudinalmente en resina y se rectificaron hasta el radio crítico (a 2,34 $\mathrm{mm}$ del centro). El revelado del tamaño de grano se realizó con una mezcla de ácido pícrico y ácido acético. Las micrografías se obtuvieron en un microscopio óptico OLYMPUS modelo BH-2-UMA. La determinación del tamaño de grano se realizó por dos procedimientos: uno, descrito en la Norma ASTM E-112 y UNE 7-280 y, el otro, por procedimientos probabilísticos basados en las medidas de las áreas de los granos mencionados en la introducción ${ }^{[12]}$.

\section{RESULTADOS Y DISCUSIÓN}

Se ha utilizado la ecuación (1) para obtener una optimización de los resultados de torsión en la aleación AZ31. Los datos experimentales han sido presentados en una publicación reciente ${ }^{[12]}$. Los resultados de esta optimización se pueden ver en la tabla I. En esta tabla, se relacionan las variables de conformado y las funcionales dependientes de la deformación de la ecuación constitutiva. Con ambas, se van a construir los coeficientes de Zener-Hollomon, $\mathrm{Z}^{*}(\varepsilon)$ asociados a cada deformación, que constituyen elementos explicativos del control y determinación de los procesos de fluencia y de los parámetros que caracterizan la RD. Los 81 parámetros de Zener-Hollomon se muestran en la tabla II.

Los resultados obtenidos para los parámetros que caracterizan los procesos de RD se detallan en la tabla III. Los 81 parámetros característicos están en 9 condiciones de $\mathrm{T}$ y $\dot{\varepsilon}$ diferentes. La tabla IV 
Tabla I. Parámetros constitutivos de la ecuación (1), a varias deformaciones

Table I. Constitutive parameters of equation (1) at various strains

\begin{tabular}{cllllll}
\hline$\varepsilon$ & $\log (\mathbf{A})$ & $\mathbf{Q}[\mathrm{kJ} / \mathbf{m o l}]$ & $\mathbf{n}$ & $\alpha\left[\mathbf{M P a}^{-1}\right]$ & $\mathbf{F}$ & $\mathbf{R}_{\mathbf{2}}$ \\
\hline 0,1 & 26,747 & 141,422 & 3,7 & 22,267 & 9,65 & 0,62 \\
0,2 & 26,34 & 146,114 & 3,446 & 0,933 & 5,43 & 0,809 \\
0,3 & 25,709 & 143,962 & 3,293 & 19,2 & 50,33 & 0,894 \\
0,4 & 22,159 & 124,282 & 2,774 & 18,667 & 69,79 & 0,921 \\
0,47 & 21,346 & 117,946 & 2,751 & 16,8 & 92,06 & 0,939 \\
0,5 & 20,845 & 115,954 & 2,575 & 18 & 85,98 & 0,935 \\
0,6 & 19,019 & 104,682 & 2,316 & 18,533 & 95,13 & 0,941 \\
0,7 & 17,02 & 89,73 & 2,275 & 16,8 & 92,11 & 0,939 \\
0,8 & 14,962 & 72,092 & 2,29 & 13,733 & 51,541 & 0,896
\end{tabular}

Tabla II. Parámetros de Zener-Hollomon normalizados frente $\varepsilon, T \dot{\varepsilon}$ y para la aleación AZ31

Table II. Normalized Zener-Hollomon parameters as a function $\varepsilon$, T and $\dot{\varepsilon}$ for the alloy AZ31

\begin{tabular}{|c|c|c|c|c|c|c|c|c|c|c|}
\hline \multirow{2}{*}{$\dot{\varepsilon}, \mathbf{s}^{-1}$} & \multirow{2}{*}{$\mathrm{T},{ }^{\circ} \mathrm{C}$} & \multicolumn{9}{|c|}{$\ln (Z / A)$} \\
\hline & & $\varepsilon=\mathbf{0 , 1}$ & $\varepsilon=0,2$ & $\varepsilon=0,3$ & $\varepsilon=\mathbf{0 , 4}$ & $\varepsilon=0,47$ & $\varepsilon=\mathbf{0 , 5}$ & $\varepsilon=0,6$ & $\varepsilon=0,7$ & $\varepsilon=0,8$ \\
\hline 0,73 & 450 & $-3,52$ & $-2,34$ & $-2,06$ & $-1,79$ & $-2,03$ & $-1,86$ & $-1,91$ & $-2,40$ & $-3,28$ \\
\hline 0,73 & 410 & $-2,14$ & $-0,91$ & $-0,66$ & $-0,58$ & $-0,88$ & $-0,73$ & $-0,89$ & $-1,53$ & $-2,57$ \\
\hline 0,73 & 300 & 2,64 & 4,03 & 4,21 & 3,63 & 3,11 & 3,19 & 2,65 & 1,51 & $-0,14$ \\
\hline 2 & 450 & $-2,52$ & $-1,33$ & $-1,06$ & $-0,79$ & $-1,03$ & $-0,86$ & $-0,91$ & $-1,40$ & $-2,27$ \\
\hline 2 & 410 & $-1,14$ & 0,09 & 0,34 & 0,43 & 0,12 & 0,27 & 0,11 & $-0,52$ & $-1,57$ \\
\hline 2 & 300 & 3,64 & 5,03 & 5,21 & 4,63 & 4,11 & 4,19 & 3,65 & 2,51 & 0,87 \\
\hline 8,73 & 450 & $-1,04$ & 0,14 & 0,42 & 0,69 & 0,45 & 0,62 & 0,57 & 0,08 & $-0,80$ \\
\hline 8,73 & 410 & 0,33 & 1,57 & 1,82 & 1,90 & 1,60 & 1,75 & 1,59 & 0,95 & $-0,10$ \\
\hline 8,73 & 410 & 5,12 & 6,51 & 6,69 & 6,11 & 5,59 & 5,67 & 5,13 & 3,99 & 2,34 \\
\hline
\end{tabular}

Tabla III. Parámetros característicos de la RD, frente a T y $\dot{\varepsilon}$ para la aleación AZ31

Table III. Characteristic parameters of DRX against $T$ and $\dot{\varepsilon}$ for the alloy AZ31

\begin{tabular}{ccccccccccc}
\hline$\dot{\varepsilon}, \mathbf{s}^{-1}$ & T,C & $\varepsilon_{\text {is }}$ & $\varepsilon_{\text {idx }}$ & $\varepsilon_{\text {max }}$ & $\varepsilon_{\text {sat }}$ & $\varepsilon_{\text {is }}$ & $\varepsilon_{\text {idrx }}$ & $\varepsilon_{\text {max }}$ & $\varepsilon_{\text {sat }}$ & $\varepsilon_{\text {final }}$ \\
\hline 0,73 & 450 & 10,5 & 17,5 & 25,5 & 39,0 & 0,05 & 0,12 & 0,38 & 0,83 & 1,05 \\
0,73 & 410 & 21,5 & 28,5 & 39,0 & 45,5 & 0,07 & 0,14 & 0,40 & 0,58 & 1,20 \\
0,73 & 300 & 68 & 91 & 106,0 & 113 & 0,11 & 0,24 & 0,44 & 0,76 & 0,90 \\
2,00 & 450 & 16 & 24 & 32,5 & 52,5 & 0,07 & 0,17 & 0,38 & 0,78 & 0,98 \\
2,00 & 410 & 24 & 33,0 & 45,5 & 53 & 0,07 & 0,14 & 0,43 & 1,08 & 0,92 \\
2,00 & 300 & 91 & 112 & 129,0 & 145 & 0,15 & 0,28 & 0,46 & 0,98 & 0,94 \\
8,73 & 450 & 30 & 40 & 55,5 & 72,5 & 0,14 & 0,22 & 0,50 & 1,39 & 1,02 \\
8,73 & 410 & 33 & 48,5 & 65,0 & 80 & 0,11 & 0,21 & 0,47 & 1,28 & 1,02 \\
8,73 & 300 & 102 & 125 & 147,0 & 165 & 0,20 & 0,31 & 0,54 & 1,15 & 0,94 \\
\hline
\end{tabular}


Tabla IV. Valores de $\varepsilon$ para el $50 \%$ de la fracción de volumen recristalizada frente T y $\dot{\varepsilon}$

Table IV. Values of $\varepsilon$ for $50 \%$ of the recrystallized volumen fraction against $T$ and $\dot{\varepsilon}$

\begin{tabular}{cccccc}
\hline$\dot{\varepsilon}\left(\mathbf{s}^{-1}\right) / \mathrm{T}\left({ }^{\circ} \mathrm{C}\right)$ & $\mathbf{3 0 0}$ & $\mathbf{3 3 0}$ & $\mathbf{3 7 0}$ & $\mathbf{4 1 0}$ & $\mathbf{4 5 0}$ \\
\hline 0,73 & 0,68 & 0,67 & 0,65 & 0,66 & 0,61 \\
2 & 0,73 & 0,68 & 0,70 & 0,65 & 0,65 \\
8,7 & 0,72 & 0,69 & 0,72 & 0,70 & 0,68
\end{tabular}

presenta, además, valores de $\varepsilon$ para el $50 \%$ de la fracción de volumen recristalizada, en función de la temperatura y la velocidad de deformación.

La figura 2 muestra la microestructura de las nueve probetas asociadas a las nueve curvas estudiadas a rotura, según las diferentes velocidades de deformación y temperatura. Los detalles de la frecuencia y distribución de área de granos vienen dados en la literatura ${ }^{[12]}$. Los resultados obtenidos, para los cuatro valores estudiados, de los tamaños de grano final, $\Phi_{1}-\Phi_{4}$, mencionados en la introducción, frente a la temperatura y la velocidad de deformación se pueden observar en la tabla V. En la tabla se dan las condiciones finales de microestructura definidas en $[0,80] \mu \mathrm{m}$, esto es, en todo el rango de resultados, por lo que son valores promedio.

También, se han calculado estos parámetros en cada intervalo de la partición $\mathrm{P}(4)$, que se han descrito, anteriormente, como la partición elegida que mejor representa los diferentes rangos asociados a los diferentes tipos de procesos de RD. Se tienen, así, 36 parámetros, 9 curvas con cuatro valores medios de tamaño de grano, que caracterizan el tamaño medio de grano y su dependencia con la temperatura y la velocidad de deformación.

Se deben considerar, también, condiciones finales, tanto las distribuciones de frecuencia, $\mathrm{f}_{\mathrm{r}}$, como las de las fracciones de área, $f$, asociadas a los mismos intervalos global y partición $\mathrm{P}(4)$. Se dispone, por lo descrito anteriormente, de numerosos parámetros

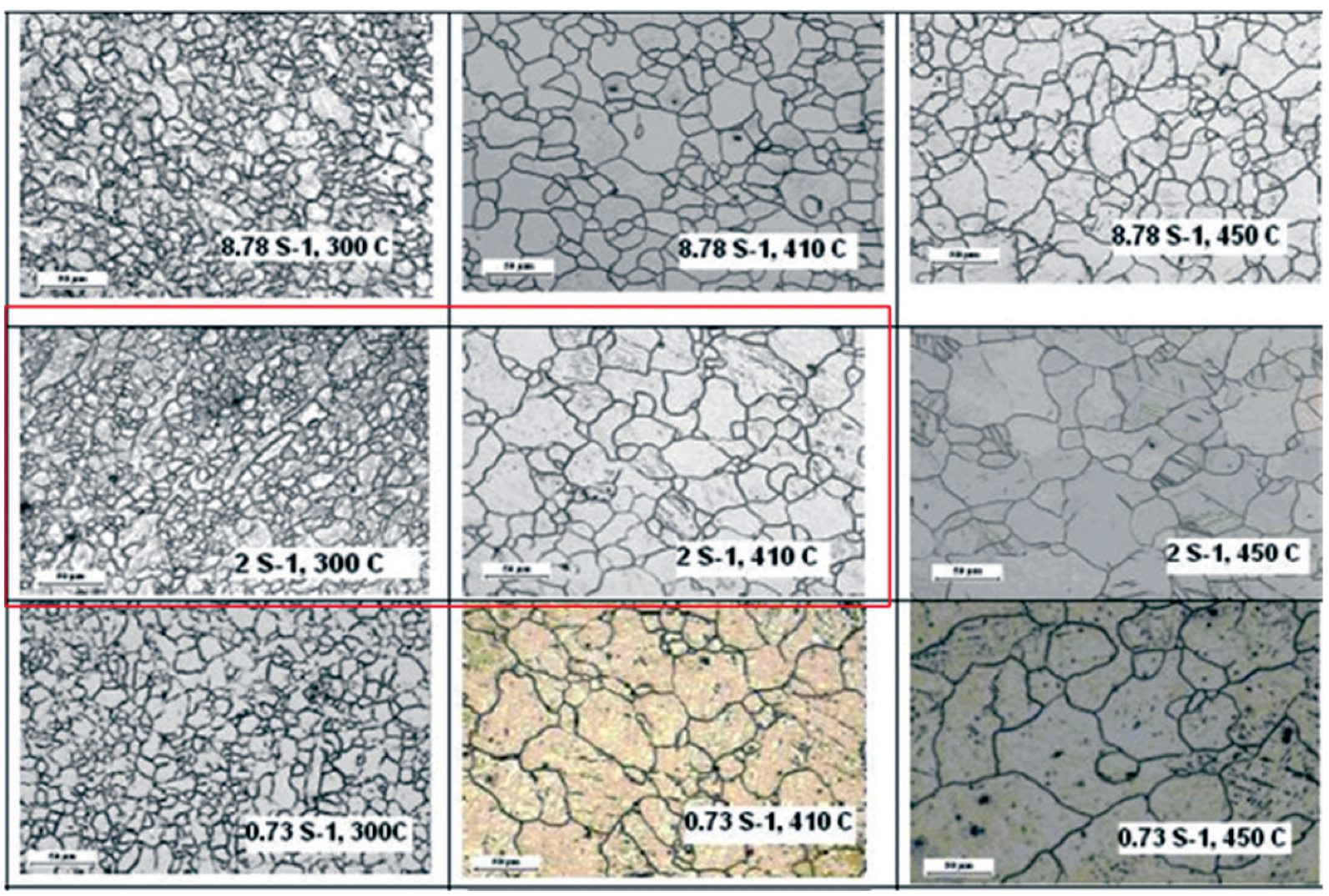

Figura 2. Micrografías de las nueve probetas asociadas a las nueve curvas estudiadas a rotura, según las diferentes velocidades de deformación y temperaturas.

Figure 2. Micrographs of nine samples associated to nine curves to rupture at various strain rates and temperatures. 
NUEVO MODELO PARA LA PREDICCIÓN DE LA MICROESTRUCTURA FNAL Y LOS PARÁMETROS DE RECRISTALIZACIÓN DINÁMICA DE MATERIALES METÁLICOS POLLCRISTALINOS. APLICACIÓN A LA ALEACIÓN DE MAGNESIO AZZ1 NEW MODEL FOR THE PREDICTION OF THE FINAL MICROSTRUCTURE AND THE DYNAMIC RECRYSTALLZATION PARAMETERS OF POLYCRYSTALLINE METALLIC MATERILLS. APPLICATION TO AZ31 MAGNESIUM ALLOY

Tabla V. Tamaños medios de grano finales, según los diferentes métodos usados, frente T y $\dot{\varepsilon}$

Table V. Average of final grain size according to the various methods used against $T$ and $\dot{\varepsilon}$

\begin{tabular}{lrrrrr}
\hline$\dot{\varepsilon}, \mathbf{s}^{-1}$ & $\mathrm{~T},{ }^{\circ} \mathrm{C}$ & $\boldsymbol{\emptyset}_{1,} \mu \mathrm{m}$ & $\boldsymbol{\emptyset}_{2,} \mu \mathrm{m}$ & $\boldsymbol{\emptyset}_{3}, \mu \mathrm{m}$ & $\boldsymbol{\emptyset}_{4,} \mu \mathrm{m}$ \\
\hline 0,73 & 450 & 30,33 & 45,10 & 46,96 & 49,23 \\
0,73 & 410 & 16,52 & 37,41 & 39,79 & 41,72 \\
0,73 & 300 & 7,35 & 10,41 & 11,18 & 12,87 \\
2 & 450 & 27,37 & 39,00 & 41,05 & 42,71 \\
2 & 410 & 17,95 & 29,90 & 30,40 & 32,51 \\
2 & 300 & 5,89 & 8,63 & 9,89 & 11,67 \\
8,73 & 450 & 15,84 & 22,61 & 22,76 & 25,39 \\
8,73 & 410 & 15,89 & 24,00 & 24,08 & 26,49 \\
8,73 & 300 & 5,40 & 9,06 & 10,19 & 12,71 \\
\hline
\end{tabular}

característicos de las distribuciones finales de microestructura del material: tamaños de grano medio, tamaños de grano asociados a intervalos de la partición $\mathrm{P}(4)$, distribuciones de frecuencias de grano en todo el rango, distribuciones de fracciones de área ocupadas en todo el rango, distribuciones de frecuencias por intervalos, según la partición $\mathrm{P}(4)$, distribuciones de fracciones de área según los intervalos de la partición $\mathrm{P}(4)$ y, todo ello, para cada combinación de $\mathrm{T}$ y descritas previamente. Como se ha trabajado con 9 curvas y con 9 combinaciones de temperatura y velocidad de deformación, además de los valores característicos de la RD expuestos anteriormente, se ha obtenido una masa de datos que es muy amplia. En la tabla VI se ofrecen 108 de estos parámetros, que se consideran especialmente relevantes. Son los valores de las frecuencias de grano, fracciones de área y tamaños medios de grano asociados a cada uno de los intervalos de la partición $\mathrm{P}(4)$.

La masa de datos para la modelización es tanamplia, que un enfoque de modelización directo sería,cuanto menos, criticable. Por lo tanto, en una primera aproximación, se ha utilizado un tratamiento estadístico previo de eliminación o screening, que ha consistido en un estudio de correlaciones bivariadas cruzadas entre todos los funcionales descritos, un análisis de componentes principales para la búsqueda de asociaciones intrínsecas de mínima varianza entre los mismos y ortogonalidad en los resultados, así como de la aplicación de un método de modelización multivariante y lineal, denominado paso a paso o de regresión por pasos (step-wise). Estas técnicas estadísticas, conocidas como de reducción de datos y de clasificación de datos, tienen como objetivo conseguir una disminución o reducción de las variables explicativas a utilizar en modelos primarios, entendiendo por tales, modelos lineales generales con máxima varianza explicada. Su objetivo no es establecer leyes sino conseguir las mejores relaciones de dependencia entre las variables a explicar y un conjunto, en general muy amplio, de variables explicativas, entre las cuales pueden darse casos de colinealidad, redundancia, etc. De entre todas ellas, se ha seleccionado el método de análisis factorial en su aplicación de componentes principales, usando la extracción de las componentes mediante los algoritmos "varimax", para la reducción y selección de

Tabla VI. Distribuciones de fracciones de área y de frecuencias frente a la temperatura y velocidad de deformación para la partición $\mathrm{P}(4)$

Table VI. Area fraction and frequency distributions against temperature and strain rate for the $P(4)$ partition

\begin{tabular}{|c|c|c|c|c|c|c|c|c|c|c|c|c|c|}
\hline \multirow[t]{2}{*}{$\varepsilon, \mathbf{s}^{-1}$} & \multirow[t]{2}{*}{$\mathrm{T},{ }^{\circ} \mathrm{C}$} & $\begin{array}{l}\text { Frac. } \\
\text { Área }\end{array}$ & $\begin{array}{l}\text { Frac. } \\
\text { Área }\end{array}$ & $\begin{array}{l}\text { Frac. } \\
\text { Área }\end{array}$ & $\begin{array}{l}\text { Frac. } \\
\text { Área }\end{array}$ & $\begin{array}{c}\text { Frecuencia, } \\
\%\end{array}$ & $\begin{array}{c}\text { Frecuencia, } \\
\%\end{array}$ & $\begin{array}{c}\text { Frecuencia, } \\
\%\end{array}$ & $\begin{array}{c}\text { Frecuencia, } \\
\%\end{array}$ & $\begin{array}{l}\text { Grano } \\
\text { medio, }\end{array}$ & $\begin{array}{l}\text { Grano } \\
\text { medio, }\end{array}$ & $\begin{array}{l}\text { Grano } \\
\text { medio, }\end{array}$ & $\begin{array}{l}\text { Grano } \\
\text { medio, }\end{array}$ \\
\hline & & {$[0,10] \mu \mathrm{m}$} & {$[10,30) \mu \mathrm{mm}$} & {$[30,50] \mu \mathrm{m}$} & {$[50,80]$ jm } & {$[0,10) \mu \mathrm{m}$} & {$[10,30] \mu \mathrm{m}$} & {$[30,50] \mu \mathrm{m}$} & {$[50,80] \mu \mathrm{m}$} & {$[0,10) \mu \mathrm{m}$} & {$[10,30) \mu \mathrm{m}$} & {$[30,50) \mathrm{\mu m}$} & {$[50,80] \mu \mathrm{m}$} \\
\hline 0,73 & & 0,42 & 15,36 & 38,58 & 45,6 & 59,52 & 47,62 & 28,57 & 14,29 & 7,32 & 19,34 & 40,41 & 62,14 \\
\hline 0,7 & 410 & 5,18 & 19,58 & 38,76 & 36,48 & 48,39 & 33,87 & 12,90 & 4,84 & 6,75 & 15,97 & 37,68 & 59,51 \\
\hline 0,73 & 300 & 35,40 & 64,60 & 0,00 & 0,00 & 77,64 & 22,36 & 0,00 & 0,00 & 5,33 & 14,38 & 0,00 & 0,00 \\
\hline 2 & 450 & 1,27 & 22,58 & 38,19 & 37,96 & 13,64 & 48,48 & 27,27 & 10,61 & 9,49 & 20,23 & 36,80 & 58,77 \\
\hline 2 & 41 & 4,18 & 37,15 & 47,35 & 11,32 & 32,48 & 50,32 & 15,29 & 1,91 & 7,42 & 17,47 & 37,40 & 52,10 \\
\hline 2 & 300 & 47,33 & 51,02 & 1,65 & 0,00 & 87,31 & 12,61 & 0,08 & 0,00 & 4,70 & 13,95 & 33,16 & 0,00 \\
\hline 8,73 & 450 & 6,21 & 68,80 & 25,00 & 0,00 & 36,79 & 57,08 & 6,13 & 0,00 & 7,22 & 19,18 & 36,50 & 0,00 \\
\hline 8,7 & 410 & 5,59 & 60,09 & 34,33 & 0,00 & 32,13 & 60,18 & 7,69 & 0,00 & 7,42 & 17,48 & 38,33 & 0,00 \\
\hline 8,73 & 300 & 40,05 & 58,39 & 1,56 & 0,00 & 85,75 & 14,17 & 0,08 & 0,00 & 3,97 & 13,90 & 30,82 & 0,00 \\
\hline
\end{tabular}


variables y el método de análisis de conglomerados o análisis "cluster". Esta metodología permite obtener los mejores $k$-means para detectar las interacciones o asociaciones intra-sujetos. En el presente caso, los sujetos son las curvas tensión-deformación. Todo el tratamiento se ha realizado con un paquete estadístico de referencia en estas técnicas que es el SPSS, versión 15.0 y con STATGRAPHICS, versión centurion $x v$. No se han detectado asociaciones en el análisis de conglomerados distintas de las previstas, temperaturas altas y bajas y velocidades de deformación altas y bajas pero, sí se han eliminado una cierta cantidad de parámetros (variables) que presentaban una cierta colinealidad. En resumen, estos procedimientos y técnicas estadísticas han permitido seleccionar las variables explicativas que mayor varianza justifican de entre aquellas que se eligieron como independientes y que son las condiciones de microestructura finales y los parámetros característicos de la RD.

Finalmente, para los tamaños de grano detallados en la tabla $\mathrm{V}$, tras los procesos de depuración y análisis estadístico, se ha procedido a modelizar relaciones funcionales estadísticas, lineales y no lineales, por métodos usuales, que postulan relaciones lineales o exponenciales entre los tamaños de grano y las funciones reducidas de Zener-Hollomon $Z^{*}$ ya que, ambos, tienen características funcionales al depender de las mismas variables T y $\dot{\varepsilon}$. Los modelos obtenidos, lineales y no lineales, tras la aplicación de las técnicas multivariantes anteriormente explicadas, han permitido seleccionar para los tamaños de grano final, un conjunto de modelos con alta significación y varianza explicada, los cuales se pueden ver resumidos en la tabla VII. En ella, se recogen tanto modelos lineales como no lineales. Hay que hacer hincapié de que se han seleccionado, solamente, aquellos cuya calidad estadística ha sido óptima.

Todos estos modelos son modelos explicativos que tienen como finalidad establecer, con alta significación, las relaciones de influencia que subyacen entre las variables resultantes del proceso o variables de interés y el conjunto de variables construidas ad-hoc en algunos casos, $Z^{*}$ de Zener-Hollomon, deducidas en el marco de una teoría, $\sigma_{\text {IDRX }}$, por ejemplo, o intrínsecas como T y $\dot{\varepsilon}$. No necesariamente, los modelos que se establecen aquí representan una ecuación con sentido o base física, sino el mejor modelo lineal general o el mejor modelo funcional, a partir de una función conocida o supuestamente aceptada para la representación de la influencia. De lo que no cabe duda, es de dos cosas: son los mejores modelos en cuanto a explicación de varianza y significación; y, posiblemente en algunos casos, sostienen procesos modelos físicos subyacentes. No se pretende aquí dar
Tabla VII. Resumen de los modelos predictivos establecidos entre los diferentes tamaños de grano finales y los parámetros de Zener-Hollomon que los optimizan a las mejores e de trabajo

Table VII. Summary of the predictive models
established among the various final grain
sizes and the Zener-Hollomon parameters that
are optimized at he best working strains

\begin{tabular}{clll}
\hline $\begin{array}{c}\text { Tamaño de } \\
\text { grano, } \mathbf{F}\end{array}$ & Modelo & $\begin{array}{c}\text { In(Z/A) } \\
\varepsilon\end{array}$ & $\mathbf{R}^{2}$ \\
\hline$\Phi_{4}$ & Exponencial & $\varepsilon=0,5$ & 0,92 \\
$\Phi_{4}$ & Lineal & $\varepsilon=0,5$ & 0,90 \\
$\Phi_{4}$ & Lineal & $\varepsilon=\max$ & 0,90 \\
$\Phi_{4}$ & Cuadrático & $\varepsilon=\max$ & 0,97 \\
$\Phi_{1}$ & Lineal & $\varepsilon=0,2$ & 0,85 \\
$\Phi_{1}$ & Cuadrático & $\varepsilon=0,2$ & 0,92 \\
$\Phi_{2}$ & Cuadrático & $\varepsilon=0,4$ & 0,97 \\
$\Phi_{3}$ & Cuadrático & $\varepsilon=0,5$ & 0,97 \\
\hline
\end{tabular}

validez a su carácter predictivo, sino, fundamentalmente, en este trabajo, exponer su capacidad explicativa y reproductiva.

Una representación de la bondad de los modelos seleccionados para el tamaño de grano $\Phi_{4}$ se ofrece en la figura 3. Entre las múltiples variables explicativas posibles, la única seleccionada para la modelización lineal ha sido la $Z^{*}$ reducida de Zener-Hollomon, asociada a la deformación $\varepsilon=0,5$. Establecido el modelo lineal por el método step-wise, entre la variable $\Phi_{4}$ y $\ln \left(Z^{*}(\varepsilon=0,5)\right)$, se han aplicado dos modelos no lineales: uno, parabólico sin ningún sentido físico y, otro, exponencial. El modelo exponencial es usual en la literatura y, como se verá posteriormente, tiene implicaciones con un mayor sentido físico. Para afrontar los pasos de la modelización no lineal, el modelo lineal general utilizado, después de los procesos de selección y depuración de la masa de datos aportados por el análisis multivariante, ha sido fundamental. En este estudio no se ha utilizado, como hacen muchos autores, la $Z^{*}$ reducida de Zener-Hollomon para la tensión de máximo o para el supuesto estado estacionario, sino, como se puede observar, se está relacionando el tamaño de grano con la $Z^{*}$ de ZenerHollomon, más adecuada.

En un proceso previo se seleccionaron los coeficientes de Zener-Hollomon para los valores de deformación, estadísticamente, más significativos. Esto tiene implicaciones posiblemente causales entre los procesos de deformación plástica acaecidos y la 

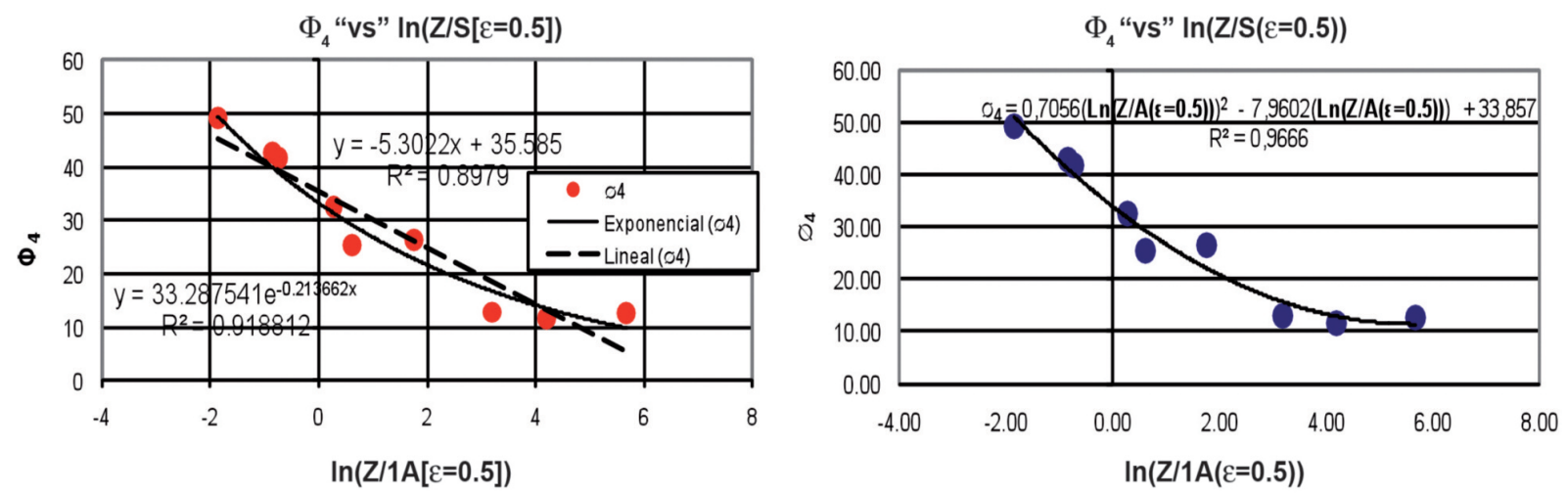

Figura 3. Modelos lineal, parabólico y exponencial para la predicción del tamaño de grano final por el método $\Phi_{4}$ frente al parámetro de Zener-Hollomon óptimo.

\section{Figure 3. Linear, parabolic and exponential models for final grain size prediction by method $\Phi_{4}$ against the optimum Zener-Hollomon parameter.}

estructura final de los granos. Es decir, existen unos modelos lineales o no lineales que permiten una modelización explicativa y predictiva de la estructura final de los tamaños de grano frente a los coeficientes de Zener-Hollomon reducidos. Si se analiza el modelo seleccionado, como óptimo para el tamaño de grano final que se ha propuesto por los métodos probabilísticos de pesado de áreas, $\Phi_{4}$, las dependencias del tamaño de grano final con las variables de conformado y con los parámetros de la ecuación constitutiva para los modelos exponencial, lineal y parabólico o cuadrático representados en la figura 3, son las siguientes:

$$
\Phi_{4}=33,2875 \cdot\left(\dot{\varepsilon} \cdot \frac{Q}{R \cdot T} e^{-0,2137}=33,2875\left\{(\sinh (0,018 \cdot \sigma))^{n}\right\}^{-0,2137}\right.
$$

Se puede observar, en la ecuación (7), que pasando la expresión de $Z^{*}$ a la forma dependiente de la tensión es posible realizar una aproximación de primer orden para la función seno hiperbólico. Se puede inferir que hay una dependencia entre el tamaño de grano y la inversa de la tensión, en una expresión aproximada a la raíz cuadrada como se puede observar en siguiente ecuación:

$$
\Phi_{4}=33,288 \cdot(0,018 \cdot \sigma)^{-0,5502775}=303,649092 \cdot \sigma^{-0,5502775}
$$

Dicho de otra forma, la ecuación (8) representa un caso general de una expresión que se puede postular para su verificación con otras aleaciones de la familia de magnesios que se están investigando y, esta relación, tendría una forma general $\Phi_{4}=\frac{\mathrm{K}}{\sqrt{\sigma_{\max }}}$.

Otro modelo se puede postular mediante la siguiente ecuación:

$$
\begin{aligned}
& \Phi_{4}=-5,3022 \cdot \ln (Z / A)+35,585= \\
& -5,3022 \cdot\left(\ln (\dot{\varepsilon})+\frac{Q}{R \cdot T}-\ln (A)\right)+35,585
\end{aligned}
$$

Aquí, se indica con claridad que la dependencia con la velocidad de deformación es débil, por su dependencia logarítmica, pero la dependencia con la temperatura es más clara. A mayor temperatura, mayor tamaño de grano, ya que $\Phi_{4} \approx \mathrm{C}_{1}-\frac{\mathrm{C}_{2}}{\mathrm{~T}}$, lo cual se puede constatar con lo mostrado en la figura 2.

Por último, se puede postular una modelización cuadrática o polinómica de la siguiente forma:

$$
\Phi_{4}=0,7056 \cdot(\ln (\mathrm{Z} / \mathrm{A}))^{2}-7,9602 \cdot \ln (\mathrm{Z} / \mathrm{A})+33,857
$$

Esta ecuación no presenta nada más que un ajuste en el rango de estudio, pero su interpretación fenomenológica es mucho más compleja.

Dado que el promedio de los máximos de las curvas estudiadas se alcanza en $\varepsilon=0,47$, hay que destacar que estas ecuaciones óptimas están calculadas para valores de deformación en máximo o próximos al máximo. Por tanto, se supone que son las condiciones del conformado del material, en este punto, las que mayor influencia tienen sobre el tamaño de grano medio final. 
A partir de las ecuaciones (7) y (9) se ha parametrizado la predicción de tamaños medios de grano final, $\Phi_{4}$, para diferentes temperaturas y velocidades de deformación. La parametrización de los modelos exponencial y lineal se pueden observar en las figuras 4 y 5 . Los modelos para la predicción de los parámetros de la RD se han estudiado de manera similar. Sus resultados óptimos se recogen en las figuras 6 a 9 .

El estudio estadístico por los métodos de regresión por pasos ha permitido seleccionar un conjunto de modelos lineales multivariantes, identificando hasta dos factores de influencia para las variables de microestructura final con una alta varianza explicada. Estos modelos no son solo predictivos sino que son, posiblemente, causales. El resumen de los parámetros que

\section{$\Phi_{4}$ VS T modelo mixto}

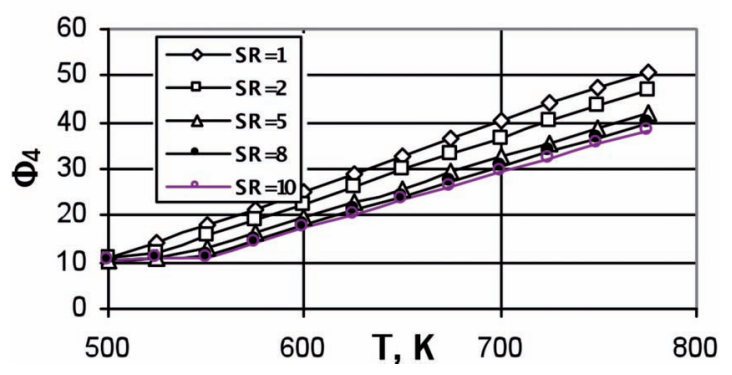

Figura 4. Modelo mixto del lineal y exponencial para la predicción de $\Phi_{4}$, parametrizados por velocidades de deformación (SR) para AZ31.

Figure 4. Mixed model of linear and exponential for the prediction of $\Phi_{4}$, parameterized by strain rates (SR) for $A Z 31$.

$$
\Phi_{4}=f(\sigma), \mu_{m}
$$

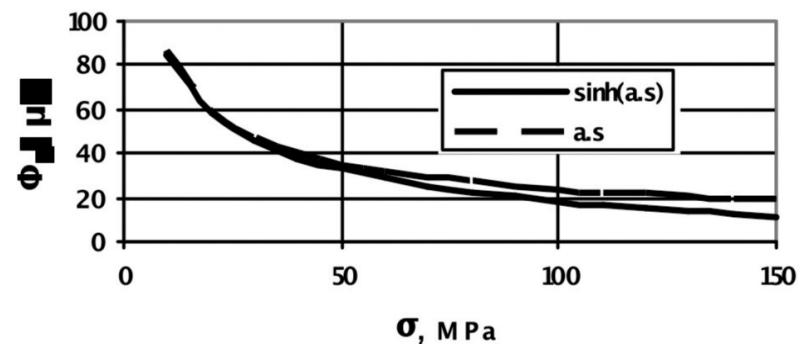

Figura 5. Comparación del modelo predictivo de $\Phi_{4}$, a partir de las ecuaciones correspondientes a la ley senh y a la ley potencial.

Figure 5. Comparison of the predictive model of $\Phi_{4}$, from the corresponding equations of sinh and power law. $\sigma_{\text {is }}$ vs $\ln (\mathrm{Z} / \mathrm{A}(\boldsymbol{\varepsilon}=0.2))$

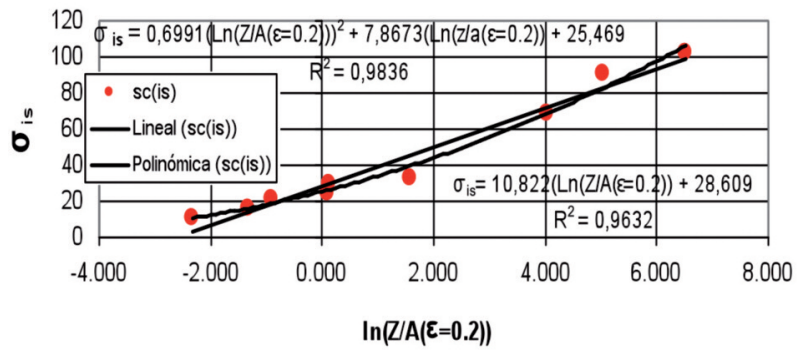

Figura 6. Modelización predictiva del valor de la tensión para el inicio de subgrano frente al parámetro de Zener-Hollomon a deformación 0,2.

Figure 6. Predictive modellization of the stress value for the start of subgrain formation against the Zener-Hollomon parameters at 0.2 strain.

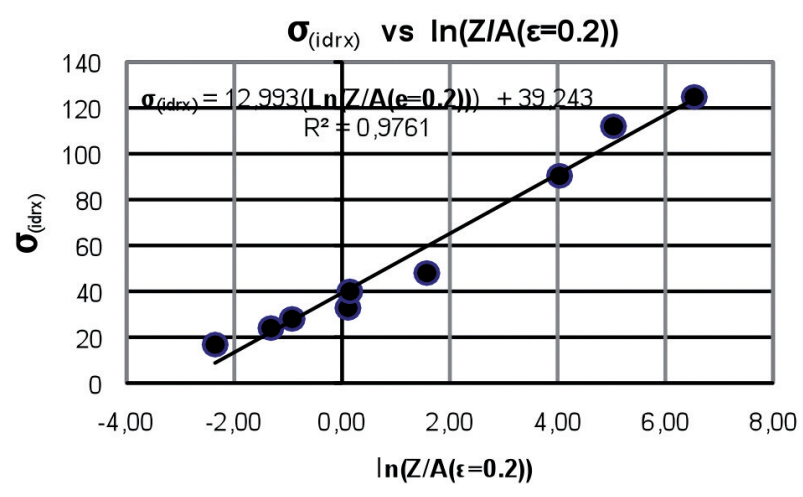

Figura 7. Modelización predictiva del valor de la tensión de inicio de la recristalización dinámica frente al mejor valor del parámetro de ZenerHollomon a deformación 0,2.

Figure 7. Predictive modellization of the stress value for the start of dynamic recrystallization against the Zener-Hollomon parameters at 0.2 strain.

definen los modelos seleccionados se puede observar en la tabla VIII.

En anteriores trabajos ${ }^{[12]}$, se han podido identificar diferentes procesos de recristalización mediante las estructuras de las distribuciones de frecuencias de grano y de fracciones de área ocupadas de los mismos. La identificación de los procesos a partir de las funciones de distribución de frecuencias y de áreas de granos permite identificar los rangos o intervalos en los que dominan los diferentes procesos identificados. Por tanto, ello orienta claramente la construcción de lo que se ha denominado partición $\mathrm{P}(4)$. 


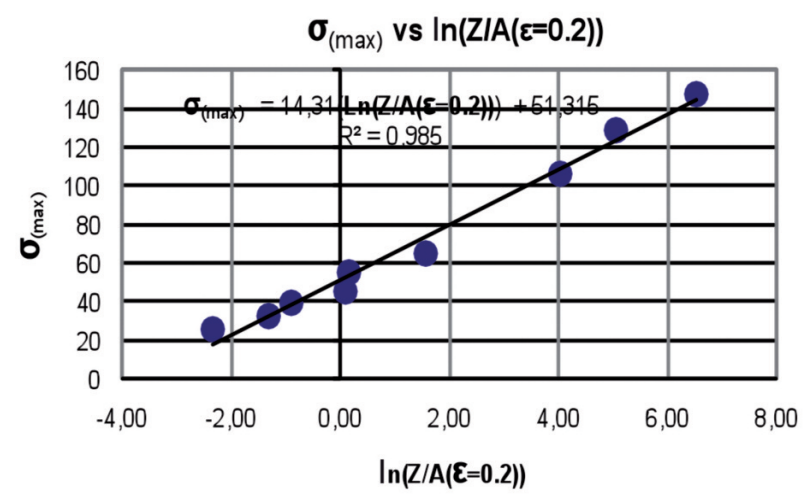

Figura 8. Modelización de los valores de la tensión de máximo a partir del parámetro de ZenerHollomon a deformación 0,2 con un 99 \% de varianza explicada.

Figure 8. Modellization of peak stress values from the Zener-Hollomon parameter at 0.2 strain with a $99 \%$ of explained variance.

Aquí, se consideran diferentes procesos de recristalización, los denominados en la literatura tipo I y tipo II. Además, hay que admitir la evolución de estos procesos. Así, se asume que, en el tipo I, hay crecimiento por absorción de collares (necklaces), dando lugar a distribuciones de grano progresivamente mayores según aumenta el número de collares desarrollados. También, hay que asumir la presencia de procesos

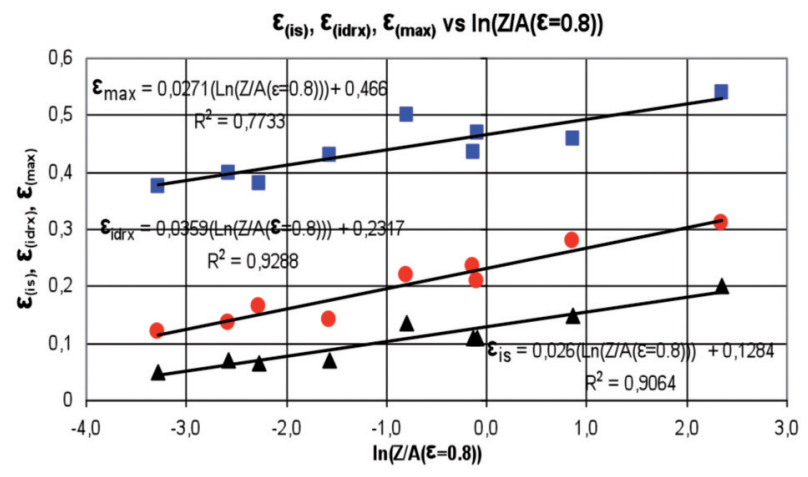

Figura 9. Diferentes modelos para la deformación de máximo, de inicio de recristalización dinámica y de inicio de formación de subgranos, en forma lineal con el valor del parámetro de Zener-Hollomon a deformación 0,8.

Figure 9. Various models for the peak strain, start of dynamic recrystallization and start of subgrain formation, in linear form, against the ZenerHollomon parameter at 0.8 strain.

secundarios o de tipo II, que tienen otro origen energético y que producen una recristalización que se suele denominar como anómala. Por lo tanto, se han realizado las fracciones de frecuencia de área ocupada y se han dividido en zonas de tamaños de grano, $[0,10),[10,30),[30,50)$ y $[50,80]$. Se ha hecho esto

Tabla VIII. Modelos óptimos alcanzados por el método de regresión multivariante por pasos para las variables que caracterizan la microestructura final de la aleación AZ31

Table VIII. Optimal models reached by the multivariant regression method by steps for the variables that characterize the final microstructure of the alloy AZ31

\begin{tabular}{|c|c|c|c|c|c|c|c|}
\hline Parámetro & Intervalo $\mu \mathrm{m}$ & Factor 1 & Factor 2 & $\mathbf{R}^{2}$ & $a_{0}$ & $a_{1}$ & $a_{2}$ \\
\hline Fracción área, \% & {$[0,10)$} & $\sigma_{\mathrm{IDRX}}$ & \multirow[t]{11}{*}{$\varepsilon^{\prime}$} & 0,986 & $-7,28$ & 0,48 & \multirow[t]{11}{*}{$-1,08$} \\
\hline Fracción área, \% & {$[10,30)$} & $\varepsilon_{\text {MAX }}$ & & 0,640 & $-94,82$ & 313,51 & \\
\hline Fracción área, \% & {$[30,50)$} & $\sigma_{\text {IDRX }}$ & & 0,850 & 50,01 & $-0,43$ & \\
\hline Fracción área, \% & {$[50,80]$} & $\ln (\mathrm{Z} / \mathrm{A})(\sigma=0,8))$ & & 0,72 & 6,85 & $-9,28$ & \\
\hline Frecuencia, \% & {$[0,10)$} & $\mathrm{T}$ & & 0,894 & 210,43 & $-0,42$ & \\
\hline Frecuencia, \% & {$[10,30)$} & $T$ & & 0,800 & $-55,60$ & 0,24 & \\
\hline Frecuencia, \% & {$[30,50)$} & $\ln (Z / A)(\varepsilon=0,5))$ & & 0,763 & 16,1 & $-3,83$ & \\
\hline Frecuencia, \% & {$[30,50)$} & $\varepsilon_{50 \% \mathrm{DRX}}$ & & 0,766 & 184,61 & $-257,19$ & \\
\hline Frecuencia, \% & {$[50,80]$} & $\varepsilon_{\text {MAX }}^{50 \% \mathrm{DRX}}$ & & 0,627 & 37,81 & $-77,36$ & \\
\hline Frecuencia, $\%$ & {$[50,80]$} & $\varepsilon_{50 \% \mathrm{DRX}}$ & & 0,687 & 83,27 & $-118,07$ & \\
\hline Grano medio, $\Phi \mu \mathrm{m}$ & {$[0,10]$} & $\sigma_{\text {Isub }}$ & & 0,815 & 8,59 & $-0,05$ & \\
\hline Grano medio, $\Phi \mu \mathrm{m}$ & {$[10,30)$} & $T^{\text {sud }}$ & \multirow{4}{*}{$\varepsilon_{\text {FINAL }}$} & 0,966 & 9,28 & 0,04 & \multirow[t]{4}{*}{$-7,93$} \\
\hline Grano medio, $\Phi \mu \mathrm{m}$ & {$[50,80]$} & $\varepsilon_{\mathrm{IDRX}}$ & & 0,77 & 107,35 & $-404,20$ & \\
\hline$\Phi_{1}$ & $\mu \mathrm{m}$ & $\ln (\mathrm{Z} / \mathrm{A})(\varepsilon=0,2))$ & & 0,85 & 19,60 & $-2,64$ & \\
\hline$\Phi_{4}$ & $\mu \mathrm{m}$ & $\ln (Z / A)(\varepsilon=0,47))$ & & 0,898 & 34,78 & $-5,23$ & \\
\hline
\end{tabular}


para frecuencias (número de granos) y para fracciones de área (área ocupada por los granos).

Hay que tener en cuenta que, por ejemplo, el valor de $\mathrm{f}[0,10)$, que representa la frecuencia de los granos que están entre 0 y 10 micrómetros, está ligado a una curva, esto es, a una temperatura y una velocidad de deformación. Por lo tanto, se tienen 9 valores de la función $\mathrm{f}[0,10)$. Igualmente, $\mathrm{f}[30,50)$ significa la fracción de área que ocupan los granos cuyo tamaño está comprendido entre 30 y 50 micrómetros, Estas funciones representan el comportamiento final del tratamiento termomecánico de la aleación que se está estudiando y, por tanto, representan el efecto que sobre estos parámetros han tenido las temperaturas y velocidades de trabajo, tras el efecto de todo el recorrido de tensiones mecánicas aplicadas a lo largo de la curva.

$\mathrm{El}$ análisis de las ecuaciones que representan los modelos que se presentan en la tabla VIII permite múltiples interpretaciones de los procesos de conformado del material. En esta tabla, hay que tener en cuenta lo siguiente:

- Un modelo lineal general multivariante tiene la forma $\mathrm{y}=\mathrm{a}_{0}+\mathrm{a}_{1} \mathrm{x}_{1}+\ldots+\mathrm{a}_{\mathrm{n}} \mathrm{x}_{\mathrm{n}}+\varepsilon$. Las columnas $\mathrm{a}_{0}, \mathrm{a}_{1}, \mathrm{a}_{2}$ aportan los coeficientes de los factores en el modelo.

- Cada uno de los factores de influencia es una variable en el modelo Factor ${ }_{i}=x_{i}$.

- Cada una de las filas es una de las variables a explicar.

- Solo se han conseguido modelos con un máximo de dos factores, que se indican como Fac 1 y Fac 2. Esto es, se han optimizado modelos con un factor y con dos factores.

- Los factores del modelo se identifican en la misma línea frente a la función a ajustar.

- La columna $\mathrm{R}^{2}$ es el coeficiente de determinación de Pearson que, multiplicado por 100, aporta el porcentaje de varianza explicada por el modelo. Cuando este valor es superior a 0,99 el modelo es muy significativo.

Así, por ejemplo, la primera fila para fracción de área \% $[0,10)$, indica que la función de $\mathrm{f}_{a}[0,10) \%$ se ha ajustado a un modelo con dos factores, que son Factor $1=\sigma_{\text {IDRX }}$ y Factor $2=\dot{\varepsilon}$. Además, se indica que el modelo no solo es óptimo (lo son todos los de la tabla) sino muy significativo, porque el valor de $\mathrm{R}^{2}=0,986$ es cercano a 0,99. Además, el modelo viene dado por $\mathrm{f}_{\mathrm{a}}[0,10) \%=-7,28+0,48 \sigma_{\text {IDRX }}-1,08 \dot{\varepsilon}$.

Estos modelos no pretenden establecer una ecuación de base física, que será otra etapa posterior de este estudio. Simplemente, aportan una información estadística clara de los factores de influencia, de su intensidad, sentido y forma de operar, independiente de otros indirectos. Son modelos explicativos y reproductivos, por lo tanto, no tienen ambición de ser predictivos, aunque lo pudieran ser.

La abundancia de modelos que se presentan en la tabla VIII requiere un proceso de análisis e investigación a desarrollar en un futuro, con las técnicas estadísticas pertinentes que, en este caso, serán las denominadas de superficies de respuesta y que permitirán integrar, mediante tales superficies (o hipersuperficies), toda la información contenida en dicha tabla.

De todos los modelos que se muestran en la tabla VIII, se quiere destacar solo algunos de ellos, por su importancia inmediata y fácil interpretación para la predicción de los tamaños de grano, en los rangos de granos más pequeños y en los rangos de granos más grandes. Se muestran las salidas de los modelos lineales generales por pasos, de alto valor de correlación y de significación. La fracción de área que ocupan los granos pequeños de los procesos de collares iniciales de la RD primaria queda determinada por la siguiente ecuación:

$$
f_{a}(\%)[0,10)=-7,279+0,478 \cdot \sigma_{i d r x}(M P a)-1,081 \cdot \dot{\varepsilon}
$$

La ecuación (11), con una $\mathrm{R}^{2}$ de 0,99, 99 \% de variabilidad explicada, muestra la relación entre la fracción de área ocupada por los granos cuyo tamaño está entre 0 y 10 um (los más pequeños) y los dos únicos parámetros que ha permitido el análisis multivariante que se relacione, que son la $\sigma_{\text {IDRX }}$ o tensión de inicio de la RD (parámetros que caracteriza a la $\mathrm{RD}$, tipo I) y $\varepsilon$. Esta, se asume derivada de los procesos de generación de collares y la velocidad de deformación, siendo la relación de la forma, ecuación (11), $\mathrm{f}_{\mathrm{a}} \%[0,10)=-7,28+0,48 \sigma_{\text {IDRX }}-1,08$, que indica que, cuanto mayor sea el valor de la tensión al cual comienza la $\mathrm{RD}$, mayor fracción de área de granos "pequeños" $[0,10)$ se consigue y, por otra parte, cuanto menor sea la velocidad de deformación mayor fracción de área de granos pequeños $[0,10)$ se consigue. Por lo tanto, como esta zona está ocupada por los granos pequeños, aquellos que se han producido en sucesivos collares, inicialmente, y que no han evolucionado rápidamente a granos mayores, son recristalizados tipo I, iniciales y tardías o iniciales antiguas no evolucionadas. Si se quiere un resultado donde dominen, se debe trabajar con velocidades de deformación bajas y conseguir que la tensión $\sigma_{\text {IDRX }}$ sea alta. Pero, por otra parte y, ligado a lo anterior, en la figura 7 se puede ver que $\sigma_{\text {IDRX }}$ tiene una relación intensa, $\mathrm{R}^{2}=0,98$, lineal directa con $\ln (\mathrm{Z} / \mathrm{A})$, esto es, $\sigma_{\mathrm{IDRX}}=\mathrm{K}+12,993 \ln (\dot{\varepsilon})+12,993 \mathrm{Q} / \mathrm{RT}$; la influencia de 12,993 no es muy intensa por el factor logarítmico (se puede unir a la constante K, para razonar) pero lo que indica el término $12,993 \mathrm{Q} / \mathrm{RT}$, es si se quieren valores altos de $\sigma_{\text {IDRX }}$ se tienen que aplicar energías de activación altas pero, lo que es más 
importante, temperaturas bajas. Así, en conclusión se puede afirmar que predominarán los procesos de RD, tipo I consiguiendo, además, que los collares iniciales no se desarrollen excesivamente, o sea, consiguiendo una fracción de área alta de granos pequeños (además, estructuralmente alineados alrededor de granos grandes) si se trabaja a temperaturas bajas y velocidades de deformación bajas.

La frecuencia de granos pequeños se ha modelizado con una explicación de varianza del $90 \%$, mediante el modelo:

$$
f_{r}(\%)[0,10)=210,432-0,422 \cdot T(K)
$$

Para conseguir abundantes granos pequeños de los primeros collares de RD se precisan, pues, temperaturas bajas, bajas $\dot{\varepsilon}$ y altas $\sigma_{\text {idrx }}$.

En el caso de granos grandes, intervalo $[50,80] \mu \mathrm{m}$, la fracción de área ocupada viene dada por:

$$
f_{a}(\%)[50,80]=6,846-9,278 \cdot \operatorname{Ln}(\mathrm{Z} / \mathrm{A \varepsilon}=0,8)
$$

Se constata la dependencia con el parámetro de Zener-Hollomon para $\varepsilon=0,8$, con un coeficiente de variación de Pearson del 72 \%, favoreciendo las temperaturas altas la presencia de procesos de RD, de tipo II. Hay que hacer hincapié en el hecho de que el funcional de Zener-Hollomon reducido, que controla la aparición de RDX de tipo II, está ligado a la deformación, 0,8 , lo que implica que para la consecución de tamaños grandes por RD de tipo II, se precisan grandes deformaciones $(0,8$ lo es para esta aleación) y altas temperaturas, con energías de activación bajas. La frecuencia de granos del mismo intervalo ocupado por granos de crecimiento anómalo viene dada por:

$$
f_{r}(\%)=83,265-118,067 \cdot \varepsilon_{50 \% R D X}
$$

Se predice, por tanto, que para conseguir numerosos granos grandes que ocupen una gran fracción de área se precisa que se alcance el $50 \%$ de la fracción de volumen recristalizado para deformaciones y tensiones de trabajo bajas.

Estos son solamente ejemplos de la capacidad de predicción que tiene el modelo propuesto en este trabajo y que puede ser de gran ayuda en la conformación de los materiales metálicos.

\section{CONCLUSIONES}

Se ha desarrollado una metodología completa integral, desde el proceso de medida, que permite modelizar las condiciones finales de microestructura de los materiales metálicos policristalinos a partir de las condiciones de trabajo y del control de los parámetros de la recristalización dinámica.

\section{Agradecimientos}

Se agradece a la CICYT la financiación de este trabajo a través de los Proyectos MAT2009-14452 y MAT2009-14385.

\section{REFERENCIAS}

[1] I. Rieiro, V. Gutiérrez, J. Castellanos, M. Carsí, M. T. Larrea y O.A. Ruano, Metal. Mater. Trans. A 41 (2010) 2.396- 2.407.

[2] I. Rieiro, M. Carsí y O.A. Ruano, Mater. Sci. Technol. 25 (2009) 995-1.002.

[3] J. Castellanos, I. Rieiro, M. El Mehtedi, M. Carsí y O.A. Ruano, Int. J. Mat. Res. 101 (2010) 787-793.

[4] V. Gutierrez, Tesis de Maestría, Universidad de Castilla La Mancha, 2009.

[5] I. Rieiro, J. Castellanos, M. Carsí, J. Muñoz, M.T. Larrea, V. Amigó y O.A. Ruano, Act. XI Cong. Nac. Tratamientos Térmicos y de Superficie, Tratermat 2008, Valencia, UPV, Valencia, España, 2008, pp.175-178.

[6] I. Rieiro, J. Castellanos, M. Carsí, D. Martín, M.T. Larrea y O. A. Ruano, Rev. Latinoam. Metal. Mater. 2 (2009) 725-734.

[7] J. Castellanos, I. Rieiro, J. Muñoz, M. Carsí y O.A. Ruano, Resúmenes XI Cong. Nac. Propiedades Mecánicas de Sólidos, El Bosque, Cádiz, España, 2008, Univ. De Cádiz, 2008, pp. 89-90.

[8] E.I Poliak y J.J. Jonas, Acta Mater. 44 (1996) 127-136.

[9] A. Galiyev, R. Kaibyshev y G. Gottstein, Acta Mater. 49 (2001) 1.199-1.207.

[10] H.J. McQueen y C.A.C. Imbert, J. Alloys Compd. 378 (2004) 35-43.

[11] I. Rieiro, J. Castellanos, M. Carsí, J. Muñoz y O.A. Ruano, Mater. Sci. Forum 604-605 (2009) 87-96.

[12] I. Rieiro, M. Larrea, J. Castellanos, M. Carsí, V. López y O.A. Ruano, Resúmenes XI Congreso Nacional de Materiales, Zaragoza, España, 2010, Sociemat, 2010, pp. 249-252.

[13] D. Ponge y G. Gottstein, Acta. Mater. 46 (1998) 69-80. 